\title{
Antitumoral effects of dovitinib in triple-negative breast cancer are synergized by calcitriol in vivo and in vitro
}

\author{
Janice García-Quiroz ${ }^{1+}$, Nohemí Cárdenas-Ochoa ${ }^{1 \dagger}$, Rocío García-Becerra² ${ }^{2}$ Gabriela Morales-Guadarrama ${ }^{1}$, Edgar \\ A. Méndez-Pérez ${ }^{1}$, Clara Santos-Cuevas ${ }^{3}$, Gerardo J. Ramírez-Nava ${ }^{3}$, Mariana Segovia-Mendoza ${ }^{4}$, Heriberto Prado- \\ Garcia $^{5}$, Euclides Avila ${ }^{1}$, Fernando Larrea ${ }^{1}$, Lorenza Díaz ${ }^{1 *}$.
}

1 Departamento de Biología de la Reproducción Dr. Carlos Gual Castro, Instituto Nacional de Ciencias Médicas y Nutrición Salvador Zubirán, Av. Vasco de Quiroga No. 15, Belisario Domínguez Sección XVI, Tlalpan 14080, Ciudad de México, México; janicegarciaq@gmail.com (J.G.-Q.); cardenas9501@gmail.com (N.C.-O.); gabriela.mguadarrama@gmail.com (G.M.-G.); edgar.mendez.p3@gmail.com (E.A.M.-P.); euclides.avilac@incmnsz.mx (E.A.); fernando.larreag@gmail.com (F.L.).

2 Departamento de Biología Molecular y Biotecnología, Instituto de Investigaciones Biomédicas, Universidad Nacional Autónoma de México, Av. Universidad 3000, Coyoacán 04510, Ciudad de México, México; rocio.garciab@iibiomedicas.unam.mx (R.G.-B.).

3 Departamento de materiales radioactivos, Instituto Nacional de Investigaciones Nucleares, Ocoyoacac 52750, Estado de México, México; clara_letici@yahoo.com.mx (C.S.-C.); gerjul5420@hotmail.com (G.J.R.-N.)

4 Departamento de Farmacología, Facultad de Medicina, Universidad Nacional Autónoma de México, Av. Universidad 3000, Coyoacán 04510, Ciudad de México, México; monaco445@yahoo.com.mx.

5 Departamento de Enfermedades Crónico-Degenerativas, Instituto Nacional de Enfermedades Respiratorias Ismael Cosío Villegas, Calzada de Tlalpan 4502, Belisario Domínguez Sección XVI, C.P. 14080, Tlalpan, Ciudad de México, México; hpradog@yahoo.com.

* Correspondence: lorenzadiaz@gmail.com.

tThese authors contributed equally to this work

\begin{abstract}
Simple Summary: The triple-negative breast cancer (TNBC) phenotype lacks a targeted therapy, is considered highly aggressive and with poor prognosis. Few therapeutic options are available for TNBC management, including chemotherapy; however, patients may develop significant adverse events and treatment-resistance. Thus, improving anticancer drugs' efficacy while reducing undesirable effects are key targets of current research. Drug combination strategies have shown to be a good therapeutic approach to address this issue, by allowing dose reduction and decreased toxicity. Regarding this, herein we explored whether combining dovitinib, a multi-kinase inhibitor, with calcitriol, a natural vitamin D anticancer metabolite, would enhance their antineoplastic effect while requiring less chemotherapeutic compound in a preclinical model of TNBC. We found that calcitriol synergized dovitinib anticancer activity in vitro and in vivo, allowing for a significant dovitinib dose-reduction while maintaining its antiproliferative potency. Our results may help to conceptualize further studies to provide TNBC-patients less toxic therapeutic options.
\end{abstract}

\begin{abstract}
Chemotherapy is a standard therapeutic option for triple-negative breast cancer (TNBC); however, its effectiveness is often compromised by drug-related toxicity and resistance development. Herein, we aimed to evaluate whether an improved antineoplastic effect could be achieved in vitro and in vivo in TNBC by combining dovitinib, a multi-kinase inhibitor, with calcitriol, a natural anticancer hormone. In vitro, cell proliferation and cell-cycle distribution were studied by sulforhodamine B-assays and flow cytometry. In vivo, dovitinib/calcitriol effects on tumor growth, angiogenesis and endothelium activation were evaluated in xenografted mice by caliper measures, Itgb3-immunohistochemistry and ${ }^{99} \mathrm{~m} \mathrm{Tc}_{\mathrm{C}} \mathrm{RGD}_{2}$-tumor uptake. The drug combination elicited a synergistically improved antiproliferative effect in TNBC-derived cells, which allowed a 7-fold dovitinib dose-reduction. Mechanistically, the co-treatment induced cell death and accumulation in $S$ and G2/M phases, while inhibited tumor growth to a greater extent than each compound alone. Tumor uptake of ${ }^{99 \mathrm{~m}} \mathrm{Tc}-\mathrm{RGD}_{2}$ was reduced by dovitinib, suggesting angiogenesis inhibition, which was corroborated by decreased endothelial cell growth and tumor-vessel density. In summary, calcitriol synergized dovitinib anticancer effects in vitro and in vivo, allowing for a significant dose-reduction
\end{abstract}


of dovitinib, while maintaining its antiproliferative potency. Our results suggest the beneficial convergence of independent antitumor mechanisms of dovitinib and calcitriol to inhibit TNBC-tumor growth.

Keywords: breast cancer; dovitinib; calcitriol; combination index; dose-reduction index; synergism.

\section{Introduction}

Breast cancer is the most commonly diagnosed neoplasm and the leading cause of cancer death among women worldwide [1]. According to the tumor molecular expression profile, this neoplasm has been classified mainly into four different subtypes: Luminal A, luminal B, human epidermal growth factor receptor 2 (HER-2) enriched and triplenegative (TNBC) [2]. Identifying breast cancer subtypes led to personalized treatment, including endocrine and anti-HER-2 therapy; however, the therapeutic possibilities for the TNBC-subtype are limited due to the lack of specific targets. In this regard, options targeting different tyrosine kinase receptors (RTKs) are currently underway, like those directed to members of the fibroblast growth factor receptors (FGFRs) [3]. Indeed, abnormal FGFR signaling has been reported in TNBC-tumors [4], including overamplifications that may result in FGF addiction [4-7]. Blocking the FGFR pathway in vivo has the additional benefit of reducing tumor angiogenesis, due to its involvement in endothelial activation [8]. Regarding this, dovitinib, a potent orally bioavailable RTK inhibitor (RTKI), blocks not only FGFR 1-3, but also the vascular endothelial growth factor receptor (VEGFR 1-3) and the platelet-derived growth factor receptor (PDGFR), whose signaling pathways are involved in carcinogenesis, neovascularization, invasion, and metastasis [7]. Of note, dovitinib has been shown to inhibit FGFR, VEGFR, and PDGFR in preclinical breast cancer models $[9,10]$. Although dovitinib has been generally associated with low-grade side effects such as diarrhea, nausea, vomiting, and/or headache [11], its long-term use may result in more severe adverse events and/or acquired resistance [12-14]. Concerning this, a good strategy to avoid treatment-associated toxicity and resistance is to combine dovitinib with other antineoplastic agents to block additional tumor survival pathways, allowing to reduce the dose and/or frequency of administration. In this regard, a recent RNA-sequencing data analysis undertaken to identify potential targeting therapeutic candidates for TNBC, revealed that one of the highly expressed genes in these tumors was the vitamin D receptor (VDR), encoding the target of calcitriol [15]. Calcitriol, the vitamin D most active metabolite, exert potent antineoplastic activity by modulating diverse signaling networks involved in inhibition of cell proliferation, anti-inflammatory effects, acquisition of a more differentiated phenotype and induction of apoptosis [16]. Moreover, calcitriol has been shown to increase the sensitivity of tumor cells to various chemotherapeutic agents [17-21], with the added benefit of being a natural compound derived from dietary sources or by sun exposure. Notably, low vitamin D serum levels have been shown to correlate with increased risk of certain neoplasms, including breast cancer [22]. Therefore, herein we explored whether an improved in vitro and in vivo antineoplastic effect could be achieved in TNBC by combining dovitinib with calcitriol. We also used a vascular endothelial cell line as a control for endothelial activation.

\section{Materials and Methods}

\subsection{Cell culture}

In this study, we used the TNBC cell line MBCDF-Tum (MBCDF-T) [23], and the human endothelial cell line EA.hy926 (ATCC CRL-292, Manassas VA). The cells were main- 
tained under standard cell culture conditions. All experimental procedures were performed in DMEM-F12 medium supplemented with 100 units/mL penicillin plus 100 $\mu \mathrm{g} / \mathrm{mL}$ streptomycin and $5 \%$ charcoal-stripped-heat-inactivated fetal bovine serum.

\subsection{Proliferation studies}

Cells were seeded in 96-well plates (500-1000 cells/well) and the day after treated with

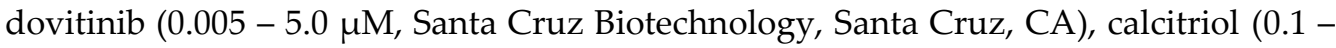
$100 \mathrm{nM}$, Sigma-Aldrich, St Louis, MO) or their respective vehicles (water or ethanol $0.1 \%$ ) for 6 days. Afterward, cell proliferation was evaluated by the sulforhodamine B colorimetric assay, as previously described [24]. Absorbance was read at $492 \mathrm{~nm}$ in a microplate reader (Synergy HT Multi-Mode Microplate Reader, BioTek, VT, USA). The concentration values that inhibited cell proliferation at $20 \%\left(\mathrm{IC}_{20}\right)$ and $50 \%\left(\mathrm{IC}_{50}\right)$ were calculated by the dose-response fitting function, using the scientific plotting software Origin 9.0 (OriginLab Corporation, Northampton, MA, USA).

\subsection{Combination index and dose reduction index determination}

To identify the nature of the compounds combination effect, the combination index (CI) and dose reduction index (DRI) were calculated as previously reported [25-27]. Results were evaluated considering that CI values $<1,=1$ or $>1$ depict synergistic, additive, or antagonistic effects, respectively, while synergism is subdivided into nearly additive (0.90-1.10), slight synergism (0.85-0.90), moderate synergism (0.7-0.85), synergism $(0.3-0.7)$, strong synergism $(0.1-0.3)$, and very strong synergism $(<0.1)$ [27]. On the other hand, DRI values $\leq 1$ or $>1$ indicate not favorable dose-reduction or favorable dose-reduction, respectively [27].

\subsection{Cell cycle analysis}

Flow cytometry analyses were performed using a FACS Aria II flow cytometer (Becton Dickinson, San Jose, CA, USA). Briefly, MBCDF-T cells were treated with dovitinib (IC50) and/or calcitriol (10 nM) for $72 \mathrm{~h}$. After that, the cells were harvested, washed in PBS pH 7.2, fixed in $70 \%$ ethanol, and kept at $-20^{\circ} \mathrm{C}$ until analysis. DNA staining with 7-amino-actinomycin D (BioLegend, San Diego, CA) was done as reported previously [23]. The results were analyzed using FlowJo Software (LLC, Ashland, OR, USA).

\subsection{Induction of tumors in athymic nude mice and therapeutic protocol}

Six-week-old female athymic nude mice (BALB/c homozygous, Crl:NU(NCr)-Foxn1nu, Charles River Laboratories, Wilmington, MA) were maintained under controlled temperature, humidity and $12 \mathrm{~h}$ light/dark cycles with sterile food (standard PMI 5053) and water ad libitum. Mice were randomly divided into four experimental groups $(\mathrm{N}=4$ each): 1) Control (C, $100 \mu \mathrm{L}$ sterile saline $0.9 \% \mathrm{NaCl}$ i.p. q.wk), 2) Calcitriol (Cal, 0.25 $\mu \mathrm{g} / 100 \mu \mathrm{L}$ i.p. q.wk, Geldex, GELpharma, México), 3) Dovitinib (Dov, 20 mg/kg twice a week i.p, CAS 852433-84-2, Santa Cruz), and 4) Dovitinib plus calcitriol (Dov+Cal). The treatments were initiated the next day after the subcutaneous injection of MBCDFT cells $(1.0 \times 106 / 0.1 \mathrm{~mL}$ sterile $0.9 \% \mathrm{NaCl})$ into the back of mice and were maintained for 3 weeks. To determine any toxic effect of the drug, mice were weighed three times per week. Tumor volume was calculated by caliper measures and the standard formula (length $\mathrm{x}$ width2)/2, where length and width are the largest and smallest dimension, respectively. At the end of the experiments, mice were sacrificed by cervical dislocation under anesthesia (sodium pentobarbital $80 \mathrm{mg} / \mathrm{kg}$ i.p.), and tumors were fixed in paraformaldehyde for immunohistochemical staining.

\subsection{SPECT/CT imaging}

To acquire tumor images of activated endothelium, one mouse from each group was placed in the prone position in an induction chamber and anesthetized $(2 \%$ isoflurane in $100 \%$ oxygen). Under anesthesia, an intravenous injection of $99 \mathrm{mTc}-\mathrm{RGD}_{2}$ (7.4 MBq/ $0.05 \mathrm{~mL}$, ININ, México), a marker of endothelial activation, was administered. After 3- 
$4 \mathrm{~h}$, the radiopharmaceutical tumor uptake was evaluated using a micro single-photon emission computed tomography and radiographic computed tomography (SPECT/CT) scanner (Albira, ONCOVISION; Gem Imaging S.A., Valencia, Spain). Acquisition parameters were the same as reported previously [23].

\subsection{Itgb3 immunohistochemistry and tumor vessel density evaluation}

To visualize blood vessels, formaldehyde-fixed and paraffin-embedded tumor sections placed on glass coverslips were dewaxed and rehydrated using standard protocols. Antigen retrieval was accomplished by autoclaving in retriever citrate solution (BioSB, Santa Barbara, CA, USA). Tumor slides were blocked with immunodetector peroxidase blocker (BioSB) and incubated for $1 \mathrm{~h}$ with the primary antibody anti-Integrin 3 (Itgb3 1:100, Cell Signaling Technology, Beverly, MA 13166, USA), a marker of activated endothelium. After washing, slides were sequentially incubated with Immuno-Detector Biotin-Link and Immuno-Detector HRP label (BioSB) 10 min each. Staining was completed with diaminobenzidine and slides were counterstained with hematoxylin. Images were taken with a conventional microscope. Microvessel count was undertaken by three different observers using 20X photographs and considering Itgb3-positive vessels in at least three hot spots areas of each tumor, as described previously [28].

\subsection{Statistical analysis}

Statistical differences were established by one-way ANOVA followed by appropriate post-hoc tests for multiple comparisons using a specialized software package (SigmaStat 3.5, Jandel Scientific, CA, USA). Differences were considered statistically significant at $\mathrm{P}<0.05$.

\section{Results}

\subsection{Dovitinib and calcitriol differentially regulate breast cancer and endothelial cell proliferation}

The effects of dovitinib and calcitriol on MBCDF-T and EA.hy926 cell proliferation are shown in Figure 1. As depicted, dovitinib significantly inhibited breast cancer and endothelial cell proliferation in a concentration-dependent manner (Figures 1A and 1B). On the other hand, as expected and as previously reported [23], calcitriol only inhibited MBCDF-T cell proliferation and did not affect endothelial cells growth (Figure 1C and $1 \mathrm{D}$, respectively). 
A

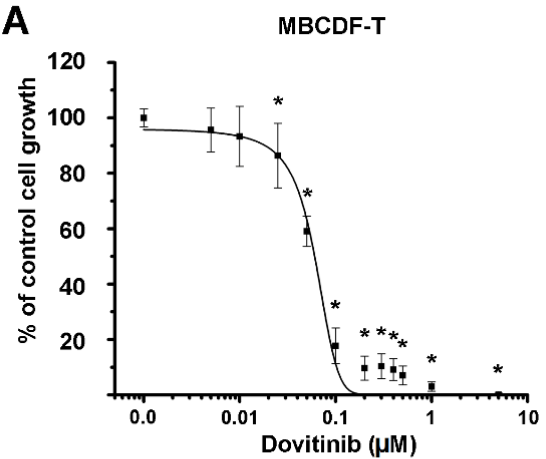

C

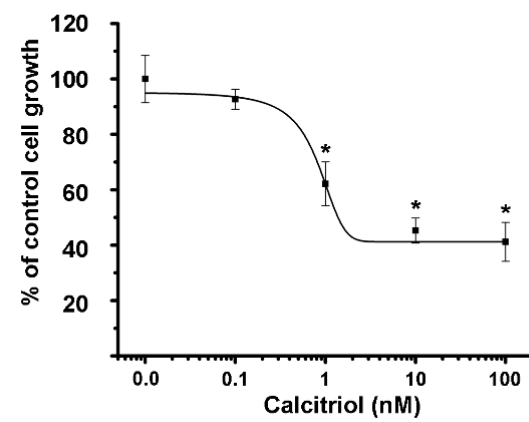

B

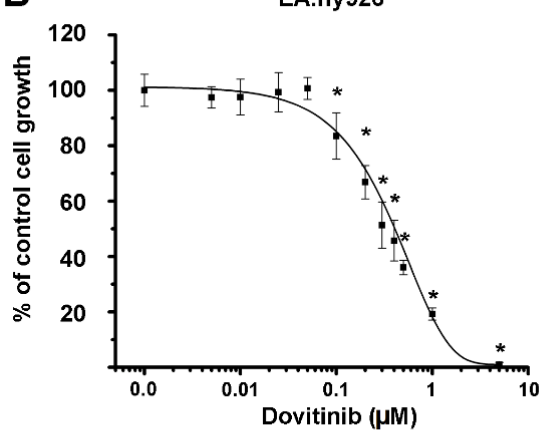

D

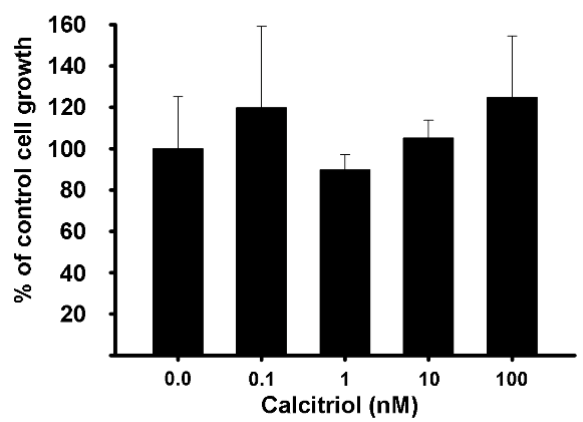

Figure 1. Dose-response curves of dovitinib and calcitriol in MBCDF-T and EA.hy926 cells. Cancer (A, C) and endothelial (B, D) cells were incubated with dovitinib (A, B) or calcitriol (C, D) for 6 days. The results are depicted as the mean \pm S.D. of at least three independent experiments by sextuplicate and were normalized vs. control values, which were set to $100 \%$. ${ }^{*} \mathrm{P}<0.001$ vs. control.

Based on the dose-response curves, $\mathrm{IC}_{20}$ and $\mathrm{IC}_{50}$ values were calculated (Table 1) and were taken into account for the combined treatments. Considering dovitinib IC 50 values, this drug inhibited more potently MBCDF-T cell growth than EA.hy926 (Table $1)$.

Table 1. IC 20 and IC 50 values of dovitinib and calcitriol in endothelial and TNBC-cells proliferation

\begin{tabular}{cllll}
\hline Cell line & \multicolumn{2}{l}{ Dovitinib (nM) } & \multicolumn{2}{l}{ Calcitriol (nM) } \\
\cline { 2 - 5 } & IC $_{20}$ & IC $_{50}$ & IC $_{20}$ & IC $_{50}$ \\
\hline MBCDF-T & 18 & 56 & 0.296 & 0.619 \\
EA.hy926 & 153 & 378 & ND & ND \\
\hline
\end{tabular}

Inhibitory concentrations at 20\% ( $\left.\mathrm{IC}_{20}\right)$ and $50 \%\left(\mathrm{IC}_{50}\right)$ were calculated based on the dose-response curves of dovitinib and calcitriol. Results are depicted as the mean of $\mathrm{N} \geq 3$ experiments. The ICs of calcitriol in EA.hy926 cells were not determined due to the lack of anti-proliferative effect of this compound in these cells (ND = Not determined).

3.2. The combination of dovitinib and calcitriol synergistically inhibited the growth of TNBCcells

Next, we sought to determine the nature of the pharmacological interaction between dovitinib and calcitriol in MBCDF-T cells. For this, the following combination schemes were evaluated (dovitinib:calcitriol): $\mathrm{IC}_{20}: \mathrm{IC}_{20}, \mathrm{IC}_{20}: \mathrm{IC}_{50}, \mathrm{IC}_{50}: \mathrm{IC}_{20}$ and IC $\mathrm{IC}_{50} \mathrm{IC} \mathrm{C}_{50}$. With all these combinations, a significantly stronger cell growth inhibitory effect was achieved than that obtained with each drug alone (Figure 2A). Notably, the calculated 
CI values showed that this effect was synergic $(\mathrm{CI}<1)$ in all cases (Table 2$)$. Then, we studied the combined effect of both drugs in EA.hy926 cells. Since calcitriol did not change endothelial growth, we tested dovitinib $\mathrm{IC}_{20}$ and $\mathrm{IC}_{50}$ values with 0.1 and $10 \mathrm{nM}$ of calcitriol. As seen in Figure 2B, calcitriol did not change dovitinib potency to inhibit endothelial cells proliferation.
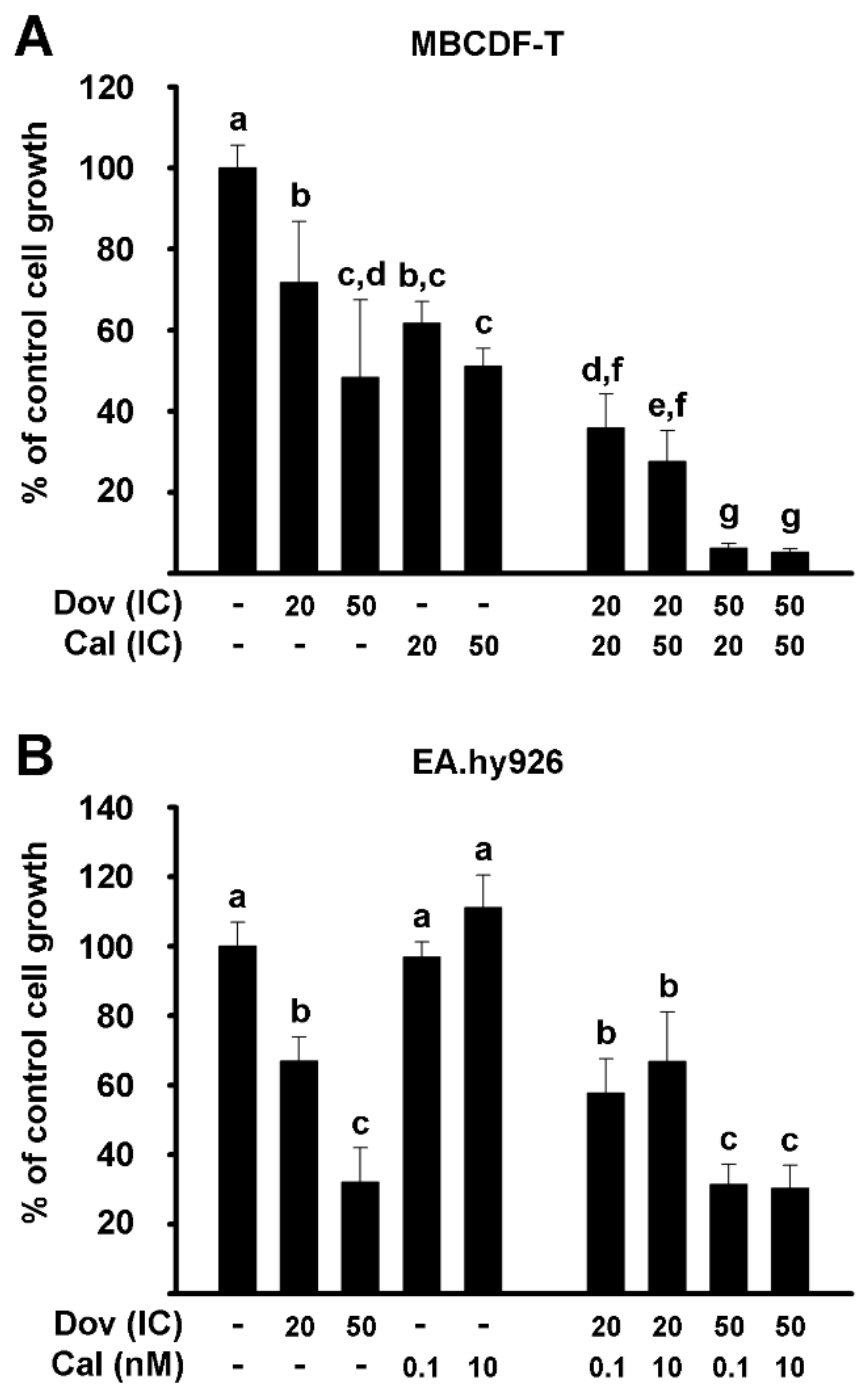

Figure 2. Calcitriol synergizes dovitinib antiproliferative activity in MBCDF-T cells. MBCDF-T cells (A) and EA.hy926 cells (B) were incubated in the presence of dovitinib (Dov), calcitriol (Cal) or their combination at their respective inhibitory concentration (IC) values at $20 \%$ or $50 \%$ during 6 days. For EA.hy 926 cells (B), Dov IC 20 and IC 50 were combined with Cal $0.1 \mathrm{nM}$ and $10 \mathrm{nM}$. Each bar represents the mean \pm S.D. of at least four independent experiments by triplicate and were normalized vs. control values, which were set to $100 \%$. Different letters indicate statistical significance $(\mathrm{P}<0.05)$.

\subsection{The combination of dovitinib with calcitriol allows for a significant dovitinib-dose reduction}

Considering the synergism elicited by the combination of dovitinib with calcitriol in tumor cells, we calculated the DRI values to determine how many folds the concentration of each compound could be reduced while maintaining the same efficacy as the drug alone (Table 2). Remarkably, in all combination schemes both dovitinib and calcitriol concentrations showed favorable DRI values (DRI $>1$ ), in accordance with the intensity of synergism (Table 2). As depicted in this table, the most favorable combination schemes were $\mathrm{IC}_{50}: \mathrm{IC}_{50}$ and $\mathrm{IC}_{50}: \mathrm{IC}_{20}$ for dovitinib:calcitriol, since dovitinib may be 
reduced up to 7 folds while calcitriol up to 28 folds, respectively. Taken together, these data show that the combination of dovitinib and calcitriol is synergistic, with favorable dose reduction values.

Table 2. Combination index values and dose reduction index for dovitinib:calcitriol treatment of MBCDF-T cells

\begin{tabular}{|c|c|c|c|c|}
\hline Combination & CI & Description & \multicolumn{2}{|c|}{ DRI (folds) } \\
\hline Dov:Cal & & & $\begin{array}{c}\text { Do } \\
\mathbf{v}\end{array}$ & Cal \\
\hline $\mathrm{IC}_{20}: \mathrm{IC}_{20}$ & 0.491 & Synergism & 4.58 & 3.66 \\
\hline $\mathrm{IC}_{20}: \mathrm{IC}_{50}$ & 0.564 & Synergism & 5.92 & 2.53 \\
\hline $\mathrm{IC}_{50}: \mathrm{IC}_{20}$ & 0.197 & Strong synergism & 6.18 & 28.62 \\
\hline $\mathrm{IC}_{50}: \mathrm{IC}_{50}$ & 0.203 & Strong synergism & 7.04 & 16.50 \\
\hline
\end{tabular}

The combination index values (CI) and dose reduction index (DRI) were calculated after co-incubating MBCDF-T cells in the presence of the inhibitory concentrations at $20 \%\left(\mathrm{IC}_{20}\right)$ and $50 \%$ ( $\left.\mathrm{IC}_{50}\right)$ of dovitinib (Dov) and calcitriol (Cal). $\mathrm{CI}<1,=1$, and $>1$ indicate synergistic, additive, or antagonistic effects, respectively. In the synergy scale, values $<0.1,0.1-0.3,0.3-0.7,0.7-0.85,0.85-0.90$, and $0.90-1.10$ indicate very strong synergism, strong synergism, synergism, moderate synergism, slight synergism, and nearly additive, respectively. DRI values $=1,>1$, and $<1$ indicate no dosereduction, favorable dose-reduction or not favorable dose-reduction, respectively, for each drug in combination. DRI values higher than 1 represent the folds of dose reduction that is allowed in combination for a given degree of effect compared with the dose of each drug alone.

\subsection{The combination of dovitinib and calcitriol promoted breast cancer cell death}

To gain insight into the mechanisms associated with the synergic antiproliferative effect of calcitriol and dovitinib, we studied the cell cycle distribution of MBCDF-T cells when exposed to both compounds alone and combined. As depicted in Figure 3, dovitinib, calcitriol and their combination significantly increased the percentage of cells in Sphase with a concomitant reduction in the percentage of cells in the G1-phase. Notably, dovitinib alone induced cell death (Sub-G1) compared to the control; however, its combination with calcitriol significantly increased this parameter. Also, the drug combination slightly but significantly augmented the percentage of cells in G2/M phases of the cell cycle (Figure 3). 


\section{Percentage of cells in each cell cycle-phase}

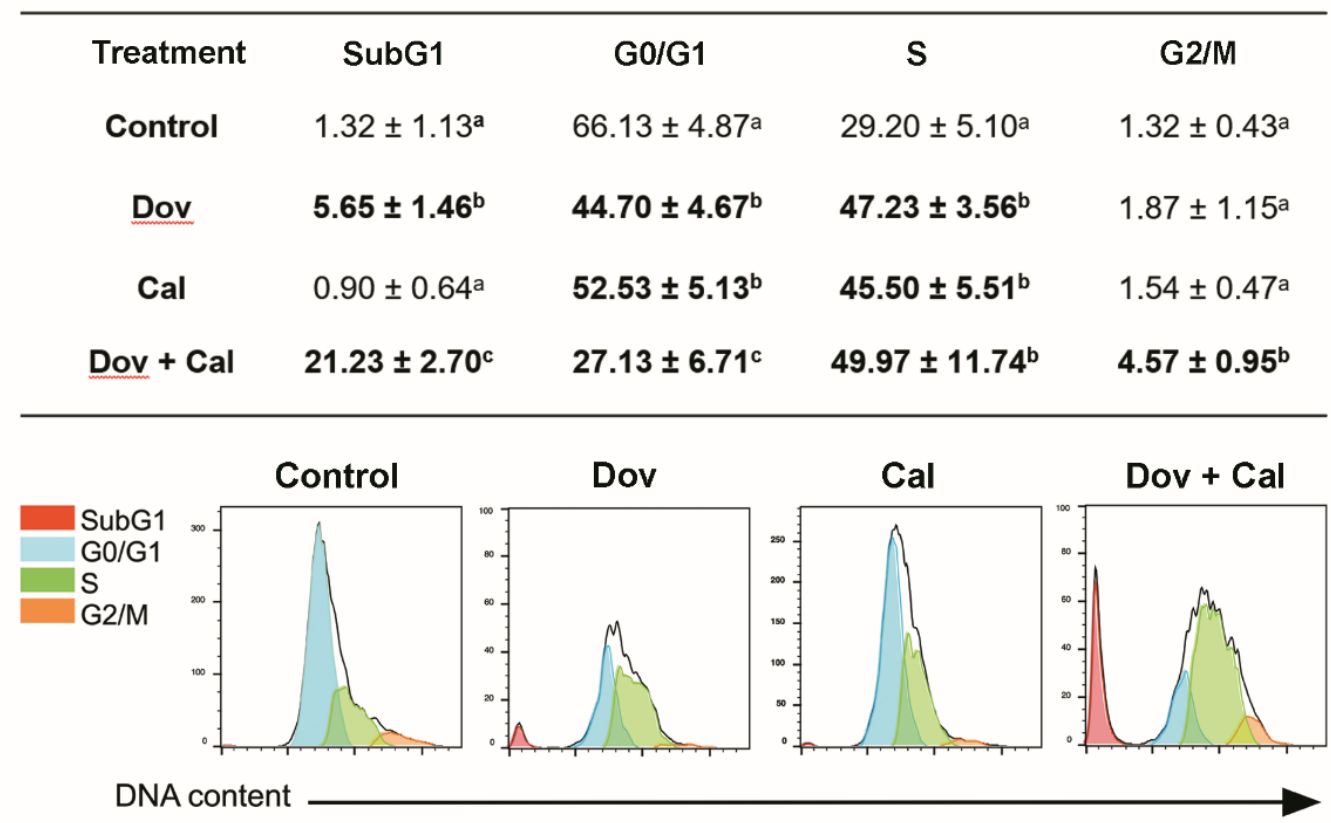

Figure 3. Modification of cell cycle distribution by dovitinib, calcitriol, and their combination in TNBC MBCDF-T cells. The effects of dovitinib (Dov, IC50), calcitriol (Cal, $10 \mathrm{nM}$ ), and their combination (Dov + Cal) on cell cycle distribution were evaluated in MBCDF-T cells. Results are shown as the mean \pm S.D. of three independent experiments. Different letters indicate statistical significance $(\mathrm{P}<0.05)$. Representative flow cytometry plots are shown in the lower panel. Cells in G1peak are shown in blue, whereas S-region cells are shown in green and G2/M cells in orange. SubG1 subpopulation, corresponding to dead cells, is shown in red.

3.5. In vivo co-administration of dovitinib and calcitriol significantly decreased tumor volume in a greater extent than each compound alone.

Based on the effective antiproliferative action of calcitriol + dovitinib combination observed in vitro, we decided to evaluate the antitumor effect of this scheme in a murine model in vivo. As expected, and as previously shown for calcitriol [23], this compound and dovitinib per se slowed MBCDF-T tumor growth compared to the control group. However, the co-administration of these compounds significantly reduced tumor volume to a greater extent than each compound alone (Figure 4A). Of note, there were no apparent side effects induced by the treatments, as judged by the absence of diarrhea and weight loss, suggesting no treatment-associated toxicity at the doses tested. 


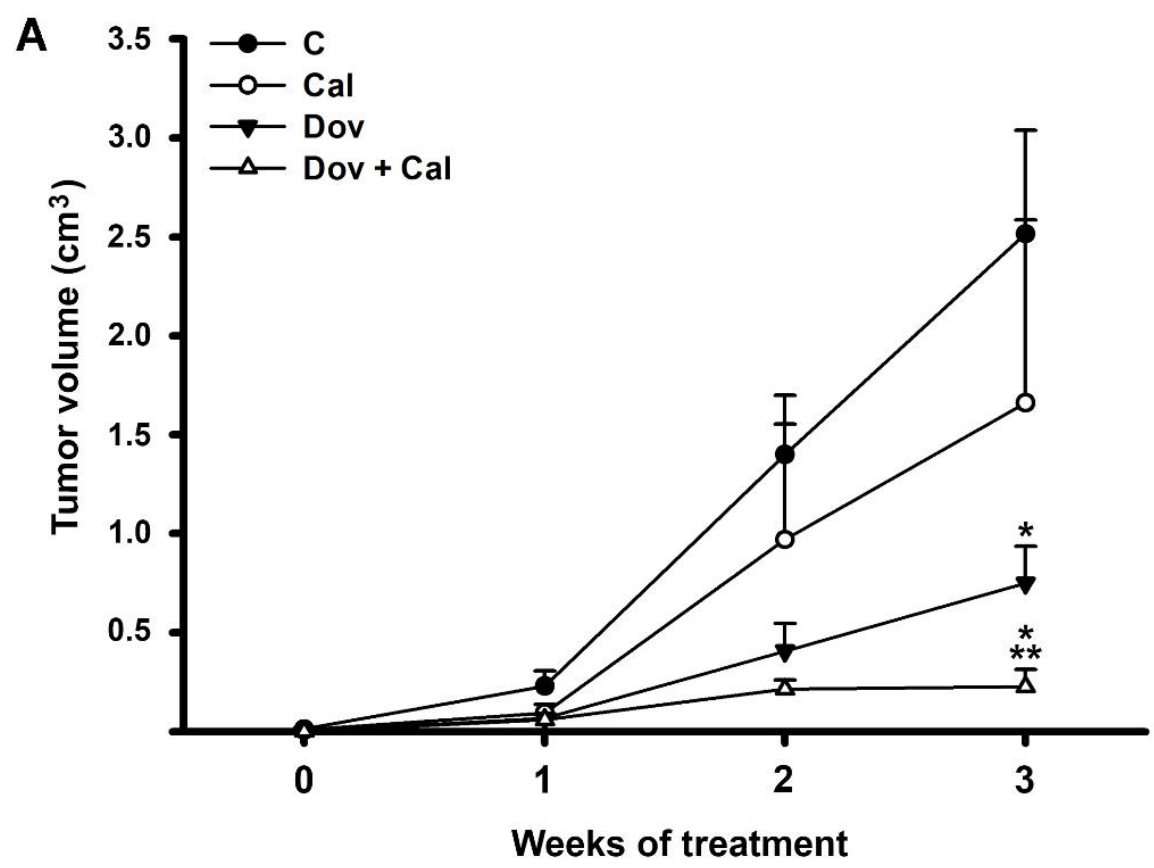

B

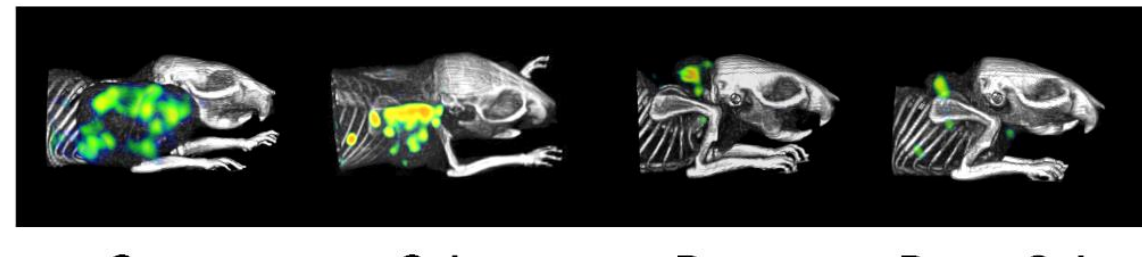

C

Cal

Dov

Dov + Cal

Figure 4. In vivo antitumor and antiangiogenic effects of dovitinib in combination with calcitriol. MBCDF-T cells were xenografted in female nude mice, starting treatments the following day with saline solution (C), calcitriol (Cal), dovitinib (Dov), or their combination (Dov + Cal) during three weeks. During the experiment, tumor volume was calculated, and the results are depicted as the mean \pm SEM (A). $\mathrm{N}=4$ mice per treatment. ${ }^{*} \mathrm{P}<0.05$ vs. $\mathrm{C},{ }^{* *} \mathrm{P}<0.05$ vs. Dov and Cal. At

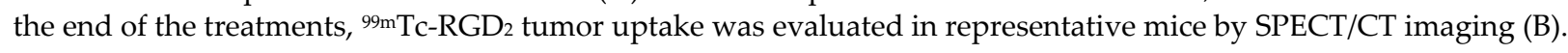

\subsection{The in vivo antiangiogenic activity of dovitinib was not affected by calcitriol}

In a representative mouse from each group, SPECT/CT images were acquired at the end of the experiment, showing decreased ${ }^{99 m T c-R G D} 2$ tumor uptake in treated mice, with a greater reduction in dovitinib and dovitinib+calcitriol groups (Figure 4B). Then, to quantitatively assess the effect of the treatments in tumor angiogenesis, vessel count was performed in Itgb3-immunostained slides. We knew from previous studies $[23,29]$ that calcitriol does not modify tumor angiogenesis in breast tumor xenografts and that its administration may increase vascular endothelial growth factor (VEGF) and FGF levels [29-31]. Therefore, we expected that the use of calcitriol with an antiangiogenic factor such as dovitinib would improve overall anticancer properties. However, it was necessary to ascertain that dovitinib antiangiogenic activity prevailed in the presence of calcitriol. As seen in Figure 5, tumors in dovitinib-treated mice significantly had a fewer number of vessels, as compared to controls. This effect was preserved in the tumors of mice co-treated with dovitinib and calcitriol (Figure 5). 


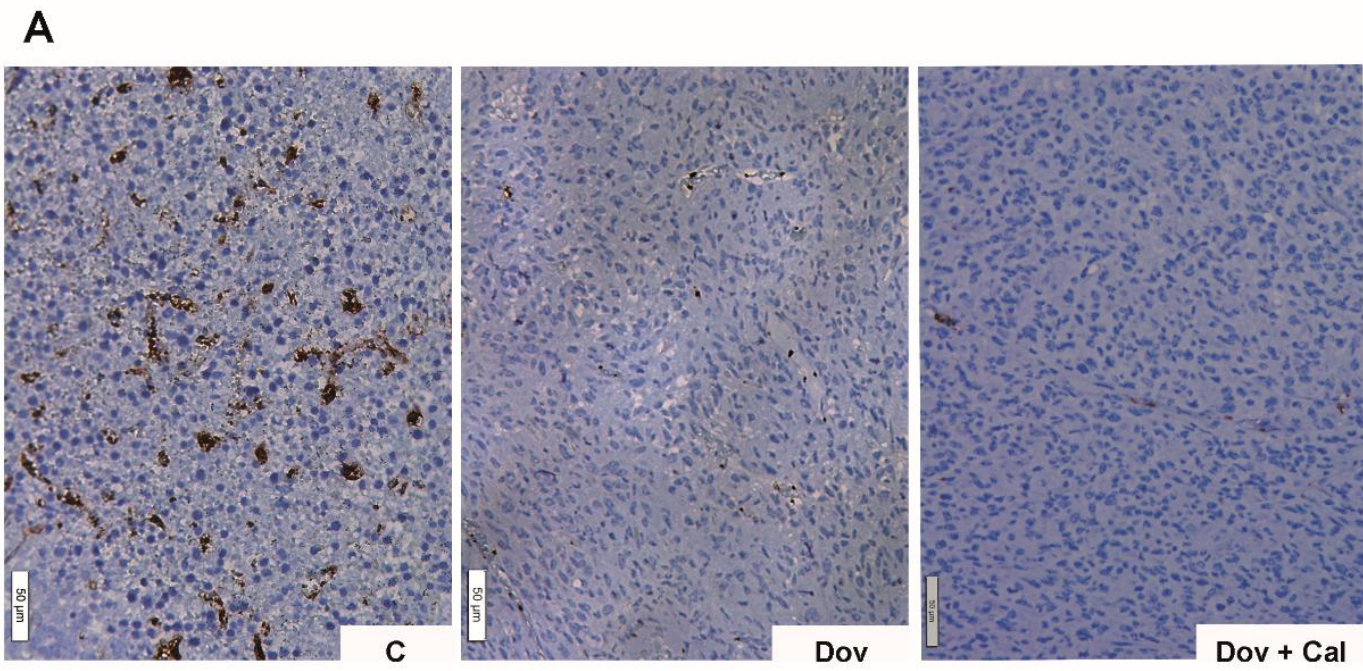

B

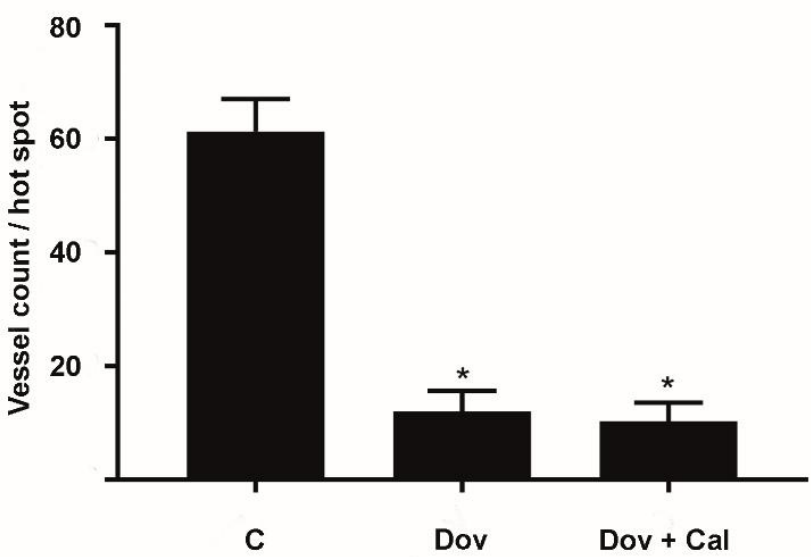

Figure 5. Vessel density was analyzed in tumors from control mice (C), treated with dovitinib (Dov), or with the combination of dovitinib and calcitriol (Dov + Cal) by Itgb3 immunostaining. (A) Representative images show Itgb3 in brown staining with 20X magnification, scale bar indicates $50 \mu \mathrm{m}$. (B) Vessel count per hot spot. Results are depicted as the mean \pm SEM of the number of vessels in three different hot spots per tumor $(\mathrm{N}=4$ different tumors/treatment), $\mathrm{P}<0.05$ vs. C.

\section{Discussion}

Due to the heterogeneous nature of TNBC, the poor prognosis, and the lack of targeted therapy, these tumors remain a clinical challenge nowadays. However, the high expression of diverse RTKs in most TNBC tumors makes these markers plausible oncological targets [32]. Drugs with multikinase inhibitory activity are a good option to treat TNBC patients, since the concomitant targeting of different RTKs translates into increased efficacy and reduced resistance. Nevertheless, some adverse side effects may develop. Here, we evaluated the in vivo and in vitro pharmacological interaction of dovitinib and calcitriol with the objective to potentiate their anticancer effect while allowing for dose reduction in TNBC. The analysis of our results using the CI theorem of Chou-Talalay [26], showed that in TNBC-cells the combination of calcitriol with dovitinib was highly synergic, reaching CI values as low as 0.2 in the $\mathrm{IC}_{50} \mathrm{IC}_{20}$ scheme. Remarkably, in all the combination schemes, the dose-reduction for each drug was favorable (DRI > 1) and was greater when the synergism was stronger. In this sense, the greatest dose-reduction was observed with the combination of dovitinib:calcitriol $\mathrm{IC}_{50}: \mathrm{IC}_{20}$ and $\mathrm{IC}_{50}: \mathrm{IC}_{50}$, where each compound concentration can be reduced by more than 7 and 28 folds, respectively. Overall, these results suggest the possibility to reduce the compounds' dose and therefore toxicity and resistance in therapeutic applications. 
To gain mechanistic insight into the drug synergism, we evaluated the effect of the combined treatment on the cell cycle distribution, and found that it strongly induced cell dead in TNBC-cells (Sub-G1 cells). Interestingly, we also found a significant accumulation of cells in the S-phase of the cell cycle in cells treated with all compounds alone and combined. Accumulation of cells in the S-phase may suggest DNA damage or inhibition of the DNA-replication machinery. Indeed, some RTKIs may directly interact with the DNA, inhibiting cancer cell proliferation [33]. Particularly in the case of dovitinib, its antiproliferative activity results not only from inhibiting multiple kinases, but also, in part, from its ability to block the ATP binding site of topoisomerases [34], which are DNA-interacting enzymes essential for proliferating cells [35]. Of note, calcitriol or its analogs have shown to enhance the efficacy of topoisomerase-inhibitors in cancer cells, as well as to induce breast cancer cell apoptosis [36,37]. Moreover, the flow cytometric analysis of our data also indicated that the dovitinib-calcitriol combination significantly increased the percentage of G2/M-cells, which may suggest mitotic catastrophe, a process associated to cell death. This phenomenon has been previously demonstrated in diverse cancer cell lines, where dovitinib promoted a delay in mitotic exit, causing G2 arrest by the activation of the DNA damage checkpoint [38]. Interestingly, enriching the number of G2-phase cells has resulted in enhanced radiosensitivity [39]; therefore, future studies are warranted combining dovitinib and calcitriol with radiotherapy.

It is known from earlier studies that calcitriol may either favor or inhibit angiogenesis, by stimulating proangiogenic factors or downregulating some RTKs, including FGFR1 [23,29-31,40,41]. However, considering that this hormone inhibits breast cancer cell proliferation through different mechanisms [16,42], we hypothesized that its combination with a VEGFR/FGFR-targeting agent would improve overall anticancer effects. Our results showed that, while the antitumor effects of both compounds were synergistically enhanced on in vivo and in vitro conditions, the antiangiogenic activity of dovitinib remained unchanged in the presence of calcitriol, as shown in endothelial cell pro-

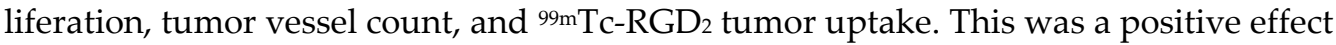
that suggests the convergence of different antitumorigenic mechanisms of calcitriol and dovitinib, resulting in a beneficial anticancer outcome. Similarly, previous studies have shown that vitamin $\mathrm{D}$ derivatives exert synergistic effects when used in combination with other oncological drugs [18-21]. In particular, it has been demonstrated that the combination of calcitriol or its analogs with gefitinib, a synthetic RTKI, enhances global anticancer activity by inhibiting tumor growth and inducing apoptosis, and in cancer patients it does not result in serious undesirable side effects $[17,20,43,44]$. To the best of our knowledge, this is the first study addressing the antineoplastic effects of dovitinib in combination with calcitriol. Notably, the effective inhibitory concentrations of calcitriol determined herein and in other studies [23], which are in the nanomolar range, are significantly lower than the blood levels reached in calcitriol-treated cancer patients in which little toxicity has been reported $[43,45]$, making it a safely achievable dose in the clinic. Likewise, the dovitinib IC $_{50}$ values calculated herein for breast cancer cells (56 $\mathrm{nM}$, equivalent to $22 \mathrm{ng} / \mathrm{mL}$ ), were significantly lower than the previously reported serum levels in dovitinib-treated patients $(60-100 \mathrm{ng} / \mathrm{mL})$ [46].

Limitations of this study include that overall survival was not evaluated as an endpoint in the in vivo murine model. This would have greatly improved the conclusions. Also, this study did not assess the mechanism by which tumors may generate escape routes after the antiangiogenic therapy, including vascular mimicry, which remain the subject of future studies.

\section{Conclusions}


In summary, we demonstrated herein that at clinically achievable and safe concentrations, the combination of calcitriol with dovitinib is highly synergic in inhibiting tumorigenesis and breast cancer cell proliferation. Mechanistically, the combination regimen inhibited tumor angiogenesis and induced cancer cell death as well as S and G2/Mphases arrest. The conclusions of this study suggest the feasibility for dose reduction to avoid dose-related toxicity while retaining therapeutic efficacy in a combined treatment scheme for TNBC in the clinic. Further studies are warranted to explore dovitinib and calcitriol in combination with radiotherapy.

Author Contributions: Conceptualization, L.D., J.G.Q., validation, J.G.Q., N.C.O., and L.D.; methodology, investigation and formal analysis, N.C.O., J.G.Q., C.S.C., G.J.R.N., M.S.M., H.P.G., G.M.G. and E.A.M.P., L.D.; writing - original draft preparation, J.G.Q. and L.D.; visualization, H.P.G., N.C.O., J.G.Q., G.M.G., C.S.C., G.J.R.N., writing-review and editing, F.L, E.A. and R.G.B.; supervision, project administration and funding acquisition, L.D. All authors have read and agreed to the published version of the manuscript.

Funding: This study was funded by Consejo Nacional de Ciencia y Tecnología de México (CONACyT), grant number A1-S-10749 to LD. The funders had no role in study design, analysis and interpretation of the data, writing of the manuscript or the decision to submit the article for publication.

Institutional Review Board Statement: The study was approved by the Internal Committee for the Care and Use of Laboratory Animals (CICUAL: BRE-1820-16/19-1) of the Instituto Nacional de Ciencias Médicas y Nutrición Salvador Zubirán, and the handling of mice was performed according to the national and international rules, including the Official Mexican Rule (NOM-062ZOO-1999).

Informed Consent Statement: Not applicable.

Data Availability Statement: Data is contained within the article or supplementary material.

Acknowledgments: We are grateful to María J Ibarra-Sánchez and José Esparza-López (Instituto Nacional de Ciencias Médicas y Nutrición Salvador Zubirán, Ciudad de México, México) for the donation of the parental cells that produced MBCDF-T cells, and to Alejandro Zentella-Dehesa (Instituto de Investigaciones Biomédicas, UNAM, México) for EA.hy926 cells donation. This study was part of the thesis work to obtain the bachelor's degree in Nutrition of NCO from Facultad de Ciencias de la Nutrición y Gastronomía de la Universidad Autónoma de Sinaloa, México.

Conflicts of Interest: The authors declare no conflict of interest.

\section{References}

1. Velloso, F.J.; Bianco, A.F.; Farias, J.O.; Torres, N.E.; Ferruzo, P.Y.; Anschau, V.; Jesus-Ferreira, H.C.; Chang, T.H.; Sogayar, M.C.; Zerbini, L.F., et al. The crossroads of breast cancer progression: insights into the modulation of major signaling pathways. OncoTargets and therapy 2017, 10, 5491-5524, doi:10.2147/OTT.S142154.

2. Perou, C.M.; Sorlie, T.; Eisen, M.B.; van de Rijn, M.; Jeffrey, S.S.; Rees, C.A.; Pollack, J.R.; Ross, D.T.; Johnsen, H.; Akslen, L.A., et al. Molecular portraits of human breast tumours. Nature 2000, 406, 747-752, doi:10.1038/35021093.

3. Santolla, M.F.; Maggiolini, M. The FGF/FGFR System in Breast Cancer: Oncogenic Features and Therapeutic Perspectives. Cancers 2020, 12, doi:10.3390/cancers12103029.

4. Chew, N.J.; Nguyen, E.V.; Su, S.P.; Novy, K.; Chan, H.C.; Nguyen, L.K.; Luu, J.; Simpson, K.J.; Lee, R.S.; Daly, R.J. FGFR3 signaling and function in triple negative breast cancer. Cell communication and signaling : CCS 2020, 18, 13, doi:10.1186/s12964-019-0486-4.

5. Pearson, A.; Smyth, E.; Babina, I.S.; Herrera-Abreu, M.T.; Tarazona, N.; Peckitt, C.; Kilgour, E.; Smith, N.R.; Geh, C.; Rooney, C., et al. High-Level Clonal FGFR Amplification and Response to FGFR Inhibition in a Translational Clinical Trial. Cancer discovery 2016, 6, 838-851, doi:10.1158/2159-8290.CD-15-1246.

6. Turner, N.; Lambros, M.B.; Horlings, H.M.; Pearson, A.; Sharpe, R.; Natrajan, R.; Geyer, F.C.; van Kouwenhove, M.; Kreike, B.; Mackay, A., et al. Integrative molecular profiling of triple negative breast cancers identifies amplicon drivers and potential therapeutic targets. Oncogene 2010, 29, 2013-2023, doi:10.1038/onc.2009.489. 
7. Yamaoka, T.; Kusumoto, S.; Ando, K.; Ohba, M.; Ohmori, T. Receptor Tyrosine Kinase-Targeted Cancer Therapy. International journal of molecular sciences 2018, 19, doi:10.3390/ijms19113491.

8. Golfmann, K.; Meder, L.; Koker, M.; Volz, C.; Borchmann, S.; Tharun, L.; Dietlein, F.; Malchers, F.; Florin, A.; Buttner, R., et al. Synergistic anti-angiogenic treatment effects by dual FGFR1 and VEGFR1 inhibition in FGFR1-amplified breast cancer. Oncogene 2018, 37, 5682-5693, doi:10.1038/s41388-018-0380-3.

9. Andre, F.; Bachelot, T.; Campone, M.; Dalenc, F.; Perez-Garcia, J.M.; Hurvitz, S.A.; Turner, N.; Rugo, H.; Smith, J.W.; Deudon, S., et al. Targeting FGFR with dovitinib (TKI258): preclinical and clinical data in breast cancer. Clinical cancer research : an official journal of the American Association for Cancer Research 2013, 19, 3693-3702, doi:10.1158/1078-0432.CCR-13-0190.

10. Issa, A.; Gill, J.W.; Heideman, M.R.; Sahin, O.; Wiemann, S.; Dey, J.H.; Hynes, N.E. Combinatorial targeting of FGF and ErbB receptors blocks growth and metastatic spread of breast cancer models. Breast cancer research : BCR 2013, 15, R8, doi:10.1186/bcr3379.

11. Musolino, A.; Campone, M.; Neven, P.; Denduluri, N.; Barrios, C.H.; Cortes, J.; Blackwell, K.; Soliman, H.; Kahan, Z.; Bonnefoi, H., et al. Phase II, randomized, placebo-controlled study of dovitinib in combination with fulvestrant in postmenopausal patients with HR(+), HER2(-) breast cancer that had progressed during or after prior endocrine therapy. Breast cancer research : BCR 2017, 19, 18, doi:10.1186/s13058-017-0807-8.

12. Corcoran, C.; O'Driscoll, L. Receptor tyrosine kinases and drug resistance: development and characterization of in vitro models of resistance to RTK inhibitors. Methods Mol Biol 2015, 1233, 169-180, doi:10.1007/978-1-4939-1789-1_16.

13. Hsiao, Y.W.; Lin, Y.C.; Hui, R.C.; Yang, C.H. Fulminant acneiform eruptions after administration of dovitinib in a patient with renal cell carcinoma. Journal of clinical oncology : official journal of the American Society of Clinical Oncology 2011, 29, e340-341, doi:10.1200/JCO.2010.32.9458.

14. Porta, C.; Giglione, P.; Liguigli, W.; Paglino, C. Dovitinib (CHIR258, TKI258): structure, development and preclinical and clinical activity. Future Oncol 2015, 11, 39-50, doi:10.2217/fon.14.208.

15. Turner, T.H.; Alzubi, M.A.; Harrell, J.C. Identification of synergistic drug combinations using breast cancer patient-derived xenografts. Scientific reports 2020, 10, 1493, doi:10.1038/s41598-020-58438-0.

16. Diaz, L.; Diaz-Munoz, M.; Garcia-Gaytan, A.C.; Mendez, I. Mechanistic Effects of Calcitriol in Cancer Biology. Nutrients 2015, 7, 5020-5050, doi:10.3390/nu7065020.

17. Jia, Z.; Zhang, Y.; Yan, A.; Wang, M.; Han, Q.; Wang, K.; Wang, J.; Qiao, C.; Pan, Z.; Chen, C., et al. 1,25-dihydroxyvitamin D3 signaling-induced decreases in IRX4 inhibits NANOG-mediated cancer stem-like properties and gefitinib resistance in NSCLC cells. Cell Death Dis 2020, 11, 670, doi:10.1038/s41419-020-02908-w.

18. Lim, S.T.; Jeon, Y.W.; Gwak, H.; Kim, S.Y.; Suh, Y.J. Synergistic anticancer effects of ruxolitinib and calcitriol in estrogen receptorpositive, human epidermal growth factor receptor 2positive breast cancer cells. Mol Med Rep 2018, 17, 5581-5588, doi:10.3892/mmr.2018.8580.

19. Maj, E.; Filip-Psurska, B.; Milczarek, M.; Psurski, M.; Kutner, A.; Wietrzyk, J. Vitamin D derivatives potentiate the anticancer and anti-angiogenic activity of tyrosine kinase inhibitors in combination with cytostatic drugs in an A549 non-small cell lung cancer model. International journal of oncology 2018, 52, 337-366, doi:10.3892/ijo.2017.4228.

20. Segovia-Mendoza, M.; Diaz, L.; Gonzalez-Gonzalez, M.E.; Martinez-Reza, I.; Garcia-Quiroz, J.; Prado-Garcia, H.; Ibarra-Sanchez, M.J.; Esparza-Lopez, J.; Larrea, F.; Garcia-Becerra, R. Calcitriol and its analogues enhance the antiproliferative activity of gefitinib in breast cancer cells. The Journal of steroid biochemistry and molecular biology 2015, 148, 122-131, doi:10.1016/j.jsbmb.2014.12.006.

21. Segovia-Mendoza, M.; Diaz, L.; Prado-Garcia, H.; Reginato, M.J.; Larrea, F.; Garcia-Becerra, R. The addition of calcitriol or its synthetic analog EB1089 to lapatinib and neratinib treatment inhibits cell growth and promotes apoptosis in breast cancer cells. Am J Cancer Res 2017, 7, 1486-1500.

22. Shaukat, N.; Jaleel, F.; Moosa, F.A.; Qureshi, N.A. Association between Vitamin D deficiency and Breast Cancer. Pak J Med Sci 2017, 33, 645-649, doi:10.12669/pjms.333.11753. 
23. Garcia-Quiroz, J.; Garcia-Becerra, R.; Santos-Cuevas, C.; Ramirez-Nava, G.J.; Morales-Guadarrama, G.; Cardenas-Ochoa, N.; Segovia-Mendoza, M.; Prado-Garcia, H.; Ordaz-Rosado, D.; Avila, E., et al. Synergistic Antitumorigenic Activity of Calcitriol with Curcumin or Resveratrol is Mediated by Angiogenesis Inhibition in Triple Negative Breast Cancer Xenografts. Cancers 2019, 11, doi:10.3390/cancers11111739.

24. Vichai, V.; Kirtikara, K. Sulforhodamine B colorimetric assay for cytotoxicity screening. Nat Protoc 2006, 1, 1112-1116, doi:10.1038/nprot.2006.179.

25. Chou, T.C. Theoretical basis, experimental design, and computerized simulation of synergism and antagonism in drug combination studies. Pharmacol Rev 2006, 58, 621-681, doi:10.1124/pr.58.3.10.

26. Chou, T.C. Drug combination studies and their synergy quantification using the Chou-Talalay method. Cancer research 2010, 70, 440-446, doi:10.1158/0008-5472.CAN-09-1947.

27. Chou, T.C. The mass-action law based algorithm for cost-effective approach for cancer drug discovery and development. $A m \mathrm{~J}$ Cancer Res 2011, 1, 925-954.

28. Weidner, N.; Semple, J.P.; Welch, W.R.; Folkman, J. Tumor angiogenesis and metastasis--correlation in invasive breast carcinoma. N Engl J Med 1991, 324, 1-8, doi:10.1056/NEJM199101033240101.

29. Garcia-Quiroz, J.; Rivas-Suarez, M.; Garcia-Becerra, R.; Barrera, D.; Martinez-Reza, I.; Ordaz-Rosado, D.; Santos-Martinez, N.; Villanueva, O.; Santos-Cuevas, C.L.; Avila, E., et al. Calcitriol reduces thrombospondin-1 and increases vascular endothelial growth factor in breast cancer cells: implications for tumor angiogenesis. The Journal of steroid biochemistry and molecular biology 2014, 144 Pt A, 215-222, doi:10.1016/j.jsbmb.2013.09.019.

30. Garcia, L.A.; Ferrini, M.G.; Norris, K.C.; Artaza, J.N. 1,25(OH)(2)vitamin D(3) enhances myogenic differentiation by modulating the expression of key angiogenic growth factors and angiogenic inhibitors in $\mathrm{C}(2) \mathrm{C}(12)$ skeletal muscle cells. The Journal of steroid biochemistry and molecular biology 2013, 133, 1-11, doi:10.1016/j.jsbmb.2012.09.004.

31. Trujillo, V.; Marin-Luevano, P.; Gonzalez-Curiel, I.; Rodriguez-Carlos, A.; Ramirez-Reyes, M.; Layseca-Espinosa, E.; EncisoMoreno, J.A.; Diaz, L.; Rivas-Santiago, B. Calcitriol promotes proangiogenic molecules in keratinocytes in a diabetic foot ulcer model. The Journal of steroid biochemistry and molecular biology 2017, 174, 303-311, doi:10.1016/j.jsbmb.2017.10.013.

32. Jansson, S.; Bendahl, P.O.; Grabau, D.A.; Falck, A.K.; Ferno, M.; Aaltonen, K.; Ryden, L. The three receptor tyrosine kinases c-KIT, VEGFR2 and PDGFRalpha, closely spaced at 4q12, show increased protein expression in triple-negative breast cancer. PLoS One 2014, 9, e102176, doi:10.1371/journal.pone.0102176.

33. Chen, K.Y.; Zhou, K.L.; Lou, Y.Y.; Shi, J.H. Exploring the binding interaction of calf thymus DNA with lapatinib, a tyrosine kinase inhibitor: multi-spectroscopic techniques combined with molecular docking. J Biomol Struct Dyn 2019, 37, 576-583, doi:10.1080/07391102.2018.1433067.

34. Hasinoff, B.B.; Wu, X.; Nitiss, J.L.; Kanagasabai, R.; Yalowich, J.C. The anticancer multi-kinase inhibitor dovitinib also targets topoisomerase I and topoisomerase II. Biochemical pharmacology 2012, 84, 1617-1626, doi:10.1016/j.bcp.2012.09.023.

35. Skok, Z.; Zidar, N.; Kikelj, D.; Ilas, J. Dual Inhibitors of Human DNA Topoisomerase II and Other Cancer-Related Targets. J Med Chem 2020, 63, 884-904, doi:10.1021/acs.jmedchem.9b00726.

36. James, S.Y.; Mercer, E.; Brady, M.; Binderup, L.; Colston, K.W. EB1089, a synthetic analogue of vitamin D, induces apoptosis in breast cancer cells in vivo and in vitro. British journal of pharmacology 1998, 125, 953-962, doi:10.1038/sj.bjp.0702103.

37. Sun, M.; Zhang, Q.; Yang, X.; Qian, S.Y.; Guo, B. Vitamin D Enhances the Efficacy of Irinotecan through miR-627-Mediated Inhibition of Intratumoral Drug Metabolism. Molecular cancer therapeutics 2016, 15, 2086-2095, doi:10.1158/1535-7163.MCT-16-0095.

38. Man, W.Y.; Mak, J.P.; Poon, R.Y. Dovitinib induces mitotic defects and activates the G2 DNA damage checkpoint. Journal of cellular and molecular medicine 2014, 18, 143-155, doi:10.1111/jcmm.12176.

39. Krueger, S.A.; Wilson, G.D.; Piasentin, E.; Joiner, M.C.; Marples, B. The effects of G2-phase enrichment and checkpoint abrogation on low-dose hyper-radiosensitivity. Int J Radiat Oncol Biol Phys 2010, 77, 1509-1517, doi:10.1016/j.ijrobp.2010.01.028. 
40. Lee, T.W.; Lee, T.I.; Lin, Y.K.; Kao, Y.H.; Chen, Y.J. Calcitriol downregulates fibroblast growth factor receptor 1 through histone deacetylase activation in HL-1 atrial myocytes. J Biomed Sci 2018, 25, 42, doi:10.1186/s12929-018-0443-3.

41. Mantell, D.J.; Owens, P.E.; Bundred, N.J.; Mawer, E.B.; Canfield, A.E. 1 alpha,25-dihydroxyvitamin D(3) inhibits angiogenesis in vitro and in vivo. Circ Res 2000, 87, 214-220, doi:10.1161/01.res.87.3.214.

42. Garcia-Becerra, R.; Diaz, L.; Camacho, J.; Barrera, D.; Ordaz-Rosado, D.; Morales, A.; Ortiz, C.S.; Avila, E.; Bargallo, E.; Arrecillas, M., et al. Calcitriol inhibits Ether-a go-go potassium channel expression and cell proliferation in human breast cancer cells. Exp Cell Res 2010, 316, 433-442, doi:10.1016/j.yexcr.2009.11.008.

43. Fakih, M.G.; Trump, D.L.; Muindi, J.R.; Black, J.D.; Bernardi, R.J.; Creaven, P.J.; Schwartz, J.; Brattain, M.G.; Hutson, A.; French, R., et al. A phase I pharmacokinetic and pharmacodynamic study of intravenous calcitriol in combination with oral gefitinib in patients with advanced solid tumors. Clinical cancer research : an official journal of the American Association for Cancer Research 2007, 13, 1216-1223, doi:10.1158/1078-0432.CCR-06-1165.

44. Muindi, J.R.; Johnson, C.S.; Trump, D.L.; Christy, R.; Engler, K.L.; Fakih, M.G. A phase I and pharmacokinetics study of intravenous calcitriol in combination with oral dexamethasone and gefitinib in patients with advanced solid tumors. Cancer Chemother Pharmacol 2009, 65, 33-40, doi:10.1007/s00280-009-1000-2.

45. Beer, T.M. Development of weekly high-dose calcitriol based therapy for prostate cancer. Urol Oncol 2003, 21, 399-405, doi:10.1016/s1078-1439(03)00170-4.

46. Wang, X.; Kay, A.; Anak, O.; Angevin, E.; Escudier, B.; Zhou, W.; Feng, Y.; Dugan, M.; Schran, H. Population pharmacokinetic/pharmacodynamic modeling to assist dosing schedule selection for dovitinib. J Clin Pharmacol 2013, 53, 14-20, doi:10.1177/0091270011433330. 